\title{
SOME GENERALIZATION PROPERTIES OF ANALYTIC FUNCTIONS CONCERNED WITH SAKAGUCHI RESULT
}

\author{
MAMORU NUNOKAWA, SHIGEYOSHI OWA, KAZUO KUROKI AND JANUSZ SOKÓŁ
}

\begin{abstract}
A subclass $\mathscr{A}(n, k)$ of analytic functions $f(z)$ in the unit disc $\mathbb{U}$ is considered. By means of the result due to K. Sakaguchi (J. Math. Soc. Japan 11(1959), $72-75$ ) for $f(z) \in \mathscr{A}(1,1)$, some generalization properties of $f(z) \in \mathscr{A}(n, k)$ with several applications are discussed.
\end{abstract}

\section{Introduction}

Let $\mathscr{H}$ denote the class of analytic functions in the unit disc $\mathbb{U}=\{z:|z|<1\}$ on the complex plane $\mathbb{C}$. Let $\mathscr{A}(n, k)$ denote the subclass of $\mathscr{H}$ which contains the functions of the form

$$
f(z)=z^{n}+\sum_{m=n+k}^{\infty} a_{m} z^{m} \quad(n \geq 1, k \geq 1),
$$

while $\mathscr{A}(1,1)=\mathscr{A}$. A set $E \subset \mathbb{C}$ is said to be starlike with respect to a point $w_{0} \in E$ if and only if the linear segment joining $w_{0}$ to every other point $w \in E$ lies entirely in $E$. An univalent function $f \in \mathscr{A}$ maps $\mathbb{U}$ onto a starlike domain $E$ with respect to a point $w_{0}=0$ if and only if

$$
\mathfrak{R e}\left\{\frac{z f^{\prime}(z)}{f(z)}\right\}>0 \quad \text { for all } z \in \mathbb{U} \text {. }
$$

Let $\mathscr{S}^{*}$ denote the class of starlike functions satisfying (1.1).

Sakaguchi [7] proved that if $f \in \mathscr{A}(1,1)$ and $g \in \mathscr{S}^{*}$, then

$$
\left\{\mathfrak{R e}\left(\frac{f^{\prime}(z)}{g^{\prime}(z)}\right)>0 \quad z \in \mathbb{U}\right\} \Rightarrow\left\{\mathfrak{R e}\left(\frac{f(z)}{g(z)}\right)>0 \quad z \in \mathbb{U}\right\} .
$$

This result found many of the applications. It was also generalized by MacGregor [3], see (2.15); and Ponnusamy and Karunakaran [6], see (2.9). In this paper we consider an extension

Received April 30, 2014, accepted July 2, 2014.

2010 Mathematics Subject Classification. Primary 30C45, Secondary 30C80.

Key words and phrases. Analytic functions, starlike functions, univalent functions, Jack's lemma, Nunokawa's lemma, Sakaguchi's condition.

Corresponding author: Janusz Sokół. 
of it too. The considered class of functions is strongly connected with the class of close-toconvex functions and with the related classes, and it is also a motivation for the studies such functions.

To prove the main results, we also need the following generalization of the Nunokawa's lemma, [4], [5].

Lemma 1.1. Let $p(z)$ be an analytic function in $|z|<1$ of the form

$$
p(z)=1+\sum_{n=k}^{\infty} a_{n} z^{n}, \quad a_{k} \neq 0,
$$

with $p(z) \neq 0$ in $|z|<1$. If there exists a point $z_{0},\left|z_{0}\right|<1$, such that

$$
|\arg \{p(z)\}|<\frac{\pi \varphi}{2} \quad \text { for } \quad|z|<\left|z_{0}\right|
$$

and

$$
\left|\arg \left\{p\left(z_{0}\right)\right\}\right|=\frac{\pi \varphi}{2}
$$

for some $\varphi>0$, then we have

$$
\frac{z_{0} p^{\prime}\left(z_{0}\right)}{p\left(z_{0}\right)}=i \ell \varphi,
$$

where

$$
\ell \geq \frac{k}{2}\left(a+\frac{1}{a}\right) \geq k \text { when } \arg \left\{p\left(z_{0}\right)\right\}=\frac{\pi \varphi}{2}
$$

and

$$
\ell \leq-\frac{k}{2}\left(a+\frac{1}{a}\right) \leq-k \text { when } \arg \left\{p\left(z_{0}\right)\right\}=-\frac{\pi \varphi}{2}
$$

where

$$
\left\{p\left(z_{0}\right)\right\}^{1 / \varphi}= \pm i a, \text { and } a>0 .
$$

The proofs of the special cases of Lemma 1.1 can be found in [1]. Also, Lemma 1.1 after suitable transformation becomes the well known Jack's lemma [2].

\section{Main results}

Theorem 2.1. Let $\alpha$ be a complex number and $\beta \leq \beta_{1}<1$. Further, let $f(z) \in \mathscr{A}(n, k)$ and $g(z) \in \mathscr{A}(n, j)(j, k \geq 1)$. Assume also that $0 \leq x<1, X \in \mathbb{R} \backslash\{0\}$ and

$$
\begin{aligned}
& \mathfrak{R e}\left\{\frac{\alpha g(z)}{z g^{\prime}(z)}\right\} \geq x \geq \frac{2\left(\beta_{1}-\beta\right)}{\left(1-\beta_{1}\right) k} \text { in }|z|<1, \\
& \mathfrak{I m}\left\{\frac{\alpha g(z)}{z g^{\prime}(z)}\right\} \geq \frac{1}{k}\left(1-\frac{X}{1-\beta_{1}}\right) \text { in }|z|<1 .
\end{aligned}
$$


Then

$$
\begin{aligned}
\mid \arg & \left\{(1-\alpha) \frac{f(z)}{g(z)}+\alpha \frac{f^{\prime}(z)}{g^{\prime}(z)}-\beta\right\} \mid \\
& <\frac{\pi}{2}+\tan ^{-1} \frac{\sqrt{k x\left(1-\beta_{1}\right)\left\{2\left(\beta-\beta_{1}\right)+\left(1-\beta_{1}\right) k x\right\}}}{|X|}
\end{aligned}
$$

implies

$$
\mathfrak{R e}\left\{\frac{f(z)}{g(z)}\right\}>\beta_{1} \text { in }|z|<1 \text {. }
$$

Proof. Let us put

$$
\lambda(z)=\frac{\alpha g(z)}{z g^{\prime}(z)}
$$

and let

$$
\begin{aligned}
p(z) & =\frac{1}{1-\beta_{1}}\left(\frac{f(z)}{g(z)}-\beta_{1}\right) \\
& =1+c_{k} z^{k}+\cdots .
\end{aligned}
$$

Then it follows that

$$
(1-\alpha) \frac{f(z)}{g(z)}+\alpha \frac{f^{\prime}(z)}{g^{\prime}(z)}-\beta=\beta_{1}-\beta+\left(1-\beta_{1}\right)\left(p(z)+\lambda(z) z p^{\prime}(z)\right) .
$$

If there exists a point $z_{0},\left|z_{0}\right|<1$, such that

$$
|\arg \{p(z)\}|<\frac{\pi}{2} \text { for }|z|<\left|z_{0}\right|
$$

and

$$
\left|\arg \left\{p\left(z_{0}\right)\right\}\right|=\frac{\pi}{2}
$$

then from Lemma 1.1, we have

$$
\frac{z_{0} p^{\prime}\left(z_{0}\right)}{p\left(z_{0}\right)}=i \ell
$$

where $p\left(z_{0}\right)= \pm i a, 0<a$ and

$$
\ell:\left\{\begin{array}{l}
\ell \geq \frac{k\left(a+\frac{1}{a}\right)}{2} \quad \text { when } \arg \left\{p\left(z_{0}\right)\right\}=\frac{\pi}{2} \\
\ell \leq-\frac{k\left(a+\frac{1}{a}\right)}{2} \text { when } \arg \left\{p\left(z_{0}\right)\right\}=-\frac{\pi}{2} .
\end{array}\right.
$$

For the case $\arg \left\{p\left(z_{0}\right)\right\}=\pi / 2$, we have

$$
\begin{aligned}
& \arg \left\{(1-\alpha) \frac{f\left(z_{0}\right)}{g\left(z_{0}\right)}+\alpha \frac{f^{\prime}\left(z_{0}\right)}{g^{\prime}\left(z_{0}\right)}-\beta\right\} \\
& \quad=\arg \left\{\beta_{1}-\beta+\left(1-\beta_{1}\right)\left(p\left(z_{0}\right)+\lambda\left(z_{0}\right) z_{0} p^{\prime}\left(z_{0}\right)\right)\right\}
\end{aligned}
$$




$$
\begin{aligned}
& =\arg \left\{p\left(z_{0}\right)\left\{(1-\beta) / p\left(z_{0}\right)+\left(1-\beta_{1}\right)\left(1+\lambda\left(z_{0}\right) z_{0} p^{\prime}\left(z_{0}\right) / p\left(z_{0}\right)\right)\right\}\right\} \\
& =\arg \left\{p\left(z_{0}\right)\left\{(1-\beta) / p\left(z_{0}\right)+\left(1-\beta_{1}\right)\left(1+\lambda\left(z_{0}\right) i \ell\right)\right\}\right\} \\
& =\arg \left\{p\left(z_{0}\right) I\left(z_{0}\right)\right\}
\end{aligned}
$$

where

$$
I\left(z_{0}\right)=-\left(\frac{\beta_{1}-\beta}{a}\right) i+\left(1-\beta_{1}\right)\left(1+\lambda\left(z_{0}\right) i \ell\right) .
$$

Then, by (2.1) and (2.7), it follows that

$$
\begin{aligned}
\mathfrak{I m}\left\{I\left(z_{0}\right)\right\} & =\mathfrak{I m}\left\{-\left(\frac{\beta_{1}-\beta}{a}\right) i+\left(1-\beta_{1}\right) \lambda\left(z_{0}\right) i \ell\right\} \\
& =\frac{\beta-\beta_{1}}{a}+\left(1-\beta_{1}\right) \ell \mathfrak{R e}\left\{\lambda\left(z_{0}\right)\right\} \\
& \geq \frac{\beta-\beta_{1}}{a}+\left(1-\beta_{1}\right) \frac{k}{2}\left(a+\frac{1}{a}\right) x .
\end{aligned}
$$

The function

$$
s(a)=\frac{\beta-\beta_{1}}{a}+\left(1-\beta_{1}\right) \frac{k}{2}\left(a+\frac{1}{a}\right) x, a>0,
$$

attains its minimum at

$$
a=\sqrt{1+\frac{2\left(\beta-\beta_{1}\right)}{\left(1-\beta_{1}\right) k x}},
$$

thus from (2.8) and after some calculation, we obtain

$$
\begin{aligned}
\mathfrak{I m}\left\{I\left(z_{0}\right)\right\} & \geq \sqrt{\left(1-\beta_{1}\right) k x\left\{2\left(\beta-\beta_{1}\right)+\left(1-\beta_{1}\right) k x\right\}} \\
& =: Y .
\end{aligned}
$$

Moreover, by (2.2), we have

$$
\begin{aligned}
\mathfrak{R e}\left\{I\left(z_{0}\right)\right\} & =\mathfrak{R e}\left\{\left(1-\beta_{1}\right)\left(1+\lambda\left(z_{0}\right) i \ell\right)\right\} \\
& =\left(1-\beta_{1}\right)\left(1-\ell \mathfrak{I m}\left\{\lambda\left(z_{0}\right)\right\}\right) \\
& \leq X .
\end{aligned}
$$

Therefore,

$$
\arg \left\{I\left(z_{0}\right)\right\} \geq \begin{cases}\tan ^{-1}(Y / X) & \text { when } X>0, \\ \pi / 2 & \text { when } X<0,\end{cases}
$$

because $I\left(z_{0}\right)$ lies in the sector

$$
\{\zeta: \mathfrak{R e} \zeta \leq X, \mathfrak{I m} \zeta \geq Y\},
$$

where $Y \geq 0$. If we use this together with $\arg \left\{p\left(z_{0}\right)\right\}=\pi / 2$, then a geometric observation gives

$$
\arg \left\{(1-\alpha) \frac{f\left(z_{0}\right)}{g\left(z_{0}\right)}+\alpha \frac{f^{\prime}\left(z_{0}\right)}{g^{\prime}\left(z_{0}\right)}-\beta\right\}
$$




$$
=\arg \left\{p\left(z_{0}\right) I\left(z_{0}\right)\right\}: \begin{cases}\geq \frac{\pi}{2}+\tan ^{-1} \frac{\sqrt{k x\left(1-\beta_{1}\right)\left\{2\left(\beta-\beta_{1}\right)+\left(1-\beta_{1}\right) k x\right\}}}{X} & \text { when } X>0, \\ \leq-\left\{\pi / 2+\tan ^{-1} \frac{\sqrt{k x\left(1-\beta_{1}\right)\left\{2\left(\beta-\beta_{1}\right)+\left(1-\beta_{1}\right) k x\right\}}}{-X}\right\} & \text { when } X<0,\end{cases}
$$

which contradicts the hypothesis (2.3). Therefore,

$$
|\arg \{p(z)\}|<\frac{\pi}{2} \text { for }|z|<1
$$

or $\mathfrak{R e}\{p(z)\}>0$, which gives (2.5).

For the case $\arg \left\{p\left(z_{0}\right)\right\}=-\pi \varphi / 2$, applying the same method as the above one, we have

$$
\begin{aligned}
& \arg \left\{(1-\alpha) \frac{f\left(z_{0}\right)}{g\left(z_{0}\right)}+\alpha \frac{f^{\prime}\left(z_{0}\right)}{g^{\prime}\left(z_{0}\right)}-\beta\right\} \\
& =\arg \left\{p\left(z_{0}\right) I\left(z_{0}\right)\right\}:\left\{\begin{array}{l}
\geq \frac{\pi}{2}+\tan ^{-1} \frac{\sqrt{k x\left(1-\beta_{1}\right)\left\{2\left(\beta-\beta_{1}\right)+\left(1-\beta_{1}\right) k x\right\}}}{X} \\
\leq-\left\{\pi / 2+\tan ^{-1} \frac{\sqrt{k x\left(1-\beta_{1}\right)\left\{2\left(\beta-\beta_{1}\right)+\left(1-\beta_{1}\right) k x\right\}}}{-X}\right\} \text { when } X>0,
\end{array}\right.
\end{aligned}
$$

This is also the contradiction and therefore it completes the proof.

Theorem 2.1 is closely related to Ponnusamy and Karunakaran's result [6, p.81] of the form

$$
\left\{\mathfrak{R e} \frac{\alpha g(z)}{z g^{\prime}(z)}>\delta \text { and } \mathfrak{R e}\left\{(1-\alpha) \frac{f(z)}{g(z)}+\alpha \frac{f^{\prime}(z)}{g^{\prime}(z)}\right\}>\beta\right\} \Rightarrow \mathfrak{R e} \frac{f(z)}{g(z)}>\frac{2 \beta+k \delta}{2+k \delta},
$$

where $\alpha$ is a complex number with $\mathfrak{R e} \alpha>0$. If we take $x=\delta$ and $\beta_{1}=(2 \beta+k \delta) /(2+k \delta)$ in Theorem 2.1, then we obtain the above Ponnusamy and Karunakaran's result under the additional assumption (2.2). But, in this special case, we can omit (2.2) in Theorem 2.1 if we let $X \rightarrow \infty$, because $\mathfrak{I m}\left\{I\left(z_{0}\right)\right\}$ becomes 0 and $\arg \left\{I\left(z_{0}\right)\right\}=0$ independently on the imaginary part of $\lambda\left(z_{0}\right)$ and of $\ell$.

For $X=1-\beta_{1}$ Theorem 2.1 provides the following corollary.

Corollary 2.2. Let $\alpha$ be a complex number and $\beta \leq \beta_{1}<1$. Further, let $f(z) \in \mathscr{A}(n, k)$ and $g(z) \in \mathscr{A}(n, j)(j, k \geq 1)$. Assume also that $0 \leq x<1$ and

$$
\begin{aligned}
& \mathfrak{R e}\left\{\frac{\alpha g(z)}{z g^{\prime}(z)}\right\} \geq x \geq \frac{2\left(\beta_{1}-\beta\right)}{\left(1-\beta_{1}\right) k} \text { in }|z|<1, \\
& \mathfrak{I m}\left\{\frac{\alpha g(z)}{z g^{\prime}(z)}\right\} \geq 0 \text { in }|z|<1 .
\end{aligned}
$$

Then

$$
\begin{aligned}
& \left|\arg \left\{(1-\alpha) \frac{f(z)}{g(z)}+\alpha \frac{f^{\prime}(z)}{g^{\prime}(z)}-\beta\right\}\right| \\
& \quad<\frac{\pi}{2}+\tan ^{-1} \sqrt{\frac{k x\left\{2\left(\beta-\beta_{1}\right)+\left(1-\beta_{1}\right) k x\right\}}{1-\beta_{1}}} \text { in }|z|<1,
\end{aligned}
$$


implies

$$
\mathfrak{R e}\left\{\frac{f(z)}{g(z)}\right\}>\beta_{1} \text { in }|z|<1
$$

If we denote

$$
\gamma=\frac{1}{k}\left(1-\frac{X}{1-\beta_{1}}\right)
$$

then Theorem 2.1 shows us the following one.

Theorem 2.3. Let $\alpha$ be a complex number and $\beta \leq \beta_{1}<1$. Further, let $f(z) \in \mathscr{A}(n, k)$ and $g(z) \in \mathscr{A}(n, j)(j, k \geq 1)$. Assume also that $0 \leq x<1, \gamma \in \mathbb{R} \backslash\{1 / k\}$ and

$$
\begin{aligned}
& \mathfrak{R e}\left\{\frac{\alpha g(z)}{z g^{\prime}(z)}\right\} \geq x \geq \frac{2\left(\beta_{1}-\beta\right)}{\left(1-\beta_{1}\right) k} \text { in }|z|<1, \\
& \mathfrak{I m}\left\{\frac{\alpha g(z)}{z g^{\prime}(z)}\right\} \geq \gamma \text { in }|z|<1 .
\end{aligned}
$$

Then

$$
\left|\arg \left\{(1-\alpha) \frac{f(z)}{g(z)}+\alpha \frac{f^{\prime}(z)}{g^{\prime}(z)}-\beta\right\}\right|<\frac{\pi}{2}+\tan ^{-1} \sqrt{\frac{k x\left\{2\left(\beta-\beta_{1}\right)+\left(1-\beta_{1}\right) k x\right\}}{\left(1-\beta_{1}\right)(1-k \gamma)^{2}}}
$$

implies

$$
\mathfrak{R e}\left\{\frac{f(z)}{g(z)}\right\}>\beta_{1} \text { in }|z|<1 \text {. }
$$

For $\beta=\beta_{1}$ Theorem 2.3 becomes the following corollary.

Corollary 2.4. Let $\alpha$ be a complex number and $\beta<1$. Further, let $f(z) \in \mathscr{A}(n, k)$ and $g(z) \in$ $\mathscr{A}(n, j)(j, k \geq 1)$. Assume also that $0 \leq x<1, \gamma \in \mathbb{R} \backslash\{1 / k\}$ and

$$
\begin{aligned}
& \mathfrak{R e}\left\{\frac{\alpha g(z)}{z g^{\prime}(z)}\right\} \geq x \geq 0 \text { in }|z|<1, \\
& \mathfrak{I m}\left\{\frac{\alpha g(z)}{z g^{\prime}(z)}\right\} \geq \gamma \text { in }|z|<1 .
\end{aligned}
$$

Then

$$
\begin{gathered}
\left|\arg \left\{(1-\alpha) \frac{f(z)}{g(z)}+\alpha \frac{f^{\prime}(z)}{g^{\prime}(z)}-\beta\right\}\right| \\
<\frac{\pi}{2}+\tan ^{-1} \frac{k x}{|1-k \gamma|}
\end{gathered}
$$

implies

$$
\mathfrak{R e}\left\{\frac{f(z)}{g(z)}\right\}>\beta \text { in }|z|<1
$$


If we put $\alpha=1, x=0$ and $\gamma \rightarrow(-\infty)$ in Corollary 2.4 then we obtain the MacGregor's result, [3], of the form

$$
\left\{\mathfrak{R e} \frac{g(z)}{z g^{\prime}(z)}>0 \text { and } \mathfrak{R e} \frac{f^{\prime}(z)}{g^{\prime}(z)}>\beta\right\} \Rightarrow \mathfrak{R e} \frac{f(z)}{g(z)}>\beta,
$$

which is a generalization of the Sakaguchi's result [7], with $\beta=0$.

Theorem 2.5. Let $h(z)$ be analytic in $|z|<1$ of the form

$$
h(z)=z+\sum_{m=k+1}^{\infty} c_{m} z^{m} .
$$

Assume also that $\beta<1$ and $n$ is a positive integer. If

$$
\left|\arg \left\{\frac{z h^{\prime}(z)}{h^{1-n}(z) z^{n}}-\beta\right\}\right|<\frac{\pi}{2}+\tan ^{-1} \frac{k}{n} \text { in }|z|<1,
$$

then

$$
\mathfrak{R e}\left\{\frac{h(z)}{z}\right\}>\beta,|z|<1
$$

Proof. Let us consider

$$
f(z)=(h(z))^{n}=z^{n}+\sum_{m=n+k}^{\infty} a_{m} z^{m}
$$

and $g(z)=z^{n}$ in Corollary 2.4. For $\alpha=1$ the conditions (2.10) and (2.11) become $1 / n \geq x, 0 \geq \gamma$ so we can put $x=1 / n$ and $\gamma=0$. Then, condition (2.12) becomes (2.16) while (2.14) becomes (2.17).

For $n=k=1$ we get the following result.

Corollary 2.6. Let $h(z)$ be analytic in $|z|<1$ of the form

$$
h(z)=z+\sum_{m=2}^{\infty} c_{m} z^{m} .
$$

Assume also that $\beta<1$. If

$$
\left|\arg \left\{h^{\prime}(z)-\beta\right\}\right|<\frac{3 \pi}{4} \text { in }|z|<1
$$

then

$$
\mathfrak{R e}\left\{\frac{h(z)}{z}\right\}>\beta,|z|<1 .
$$

Recall here another result of this type [8, p. 118] that if $h \in \mathscr{A}(1,1), \beta<1 / 3$, then

$$
\left|\arg \left\{h^{\prime}(z)\right\}\right|<\frac{\pi}{2}+\tan ^{-1} \sqrt{\frac{1-3 \beta}{1-\beta}} \text { in }|z|<1,
$$

implies

$$
\mathfrak{R e}\left\{\frac{h(z)}{z}\right\}>\beta,|z|<1 .
$$

For a related result under more complicated assumption we also refer to [9, p. 1550]. 


\section{References}

[1] S. Fukui and K. Sakaguchi, An extension of a theorem of S. Ruscheweyh, Bull. Fac. Edu. Wakayama Univ. Nat. Sci., 29(1980), 1-3.

[2] I. S. Jack, Functions starlike and convex of order $\alpha$, J. London Math. Soc., 3(1971), 469-474.

[3] T. H. MacGregor, A subordination for convex functions of order $\alpha$, J. London Math. Soc. 2(1975), 530-536.

[4] M. Nunokawa, On properties of non-Carathéodory functions, Proc. Japan Acad., 68(1992), 152-153.

[5] M. Nunokawa, On the order of strongly starlikeness of strongly convex functions, Proc. Japan Acad., 69(1993), 234-237.

[6] S. Ponnusmy and V. Karunakakaran, Differential subordination and conformal mappings, Complex variable, 11(1989), 79-86.

[7] K. Sakaguchi, On certain univalent mapping, J. Math. Soc. Japan, 11(1959), 72-75.

[8] J. Sokół, On functions with the derivative satisfying a geometric condition, Appl. Math. Comput., 204(2008), 116-119.

[9] J. Sokół, On neighborhoods of analytic functions having positive real part, Math. Nachr., 284(2011), 15471553.

Emeritus Professor, University of Gunma, Hoshikuki-cho 798-8, Chuou-Ward, Chiba, 260-0808, Japan.

E-mail: mamoru_nuno@doctor.nifty.jp

Department of Mathematics, Faculty of Education, Yamato University, Katayama 2-5-1, Suita, Osaka 564-0082, Japan.

E-mail: shige21@ican.zaq.ne.jp

Department of Mathematics, Kinki University, Higashi-Osaka, Osaka 577-8502, Japan.

E-mail: freedom@sakai.zaq.ne.jp

Department of Mathematics, Rzeszów University of Technology, Al. Powstańców Warszawy 12, 35-959 Rzeszów, Poland.

E-mail: jsokol@prz.edu.pl 